\title{
Early Detection of Prone to Failure Student Using Machine Learning Techniques
}

\author{
Prabha Siddhesh Kadam and Vinod Moreshwar Vaze \\ ${ }^{1}$ Computer Science, Shri JJT University, Rajasthan \& Assistant \\ Professor, Kirti. M. Doongursee College, Mumbai, India \\ ${ }^{2}$ Computer Science, Shri JJT University, Rajasthan, India
}

\section{ABSTRACT}

Machine learning techniques works on experience uses historical data and process them. The algorithms help to reveal facts and shows the path to move towards success. This study, uses for early detection of prone to failure using machine learning techniques. Supervised approach of machine learning used to analysis data in python colab environment. The Sample size 300 records used to evaluate data. Outcomes shows $82 \%$ accuracy with Naive Bayes classifiers. The study classifies records among three classes good, average and poor students.

KEY WORDS: MACHINE LEARNING, EARLY DETECTION, NAIVE BAYES CLASSIFIERS, LOGISTIC REGRESSION, SUPERVISED LEARNING.

\section{INTRODUCTION}

Machine learning in educational sector already marked his footprints. Adaptive learning, predictive analysis, increasing efficiency, personalized learning, learning analytics and accurately grading assignments are the few areas in the education sectors covered by this next generation technology. Every day new research and findings are coming ahead in the machine learning and deep learning, the subgroups of AI (Artificial Intelligence).

Now a day's graduation is the minimum criteria to get a good job, but the same time few students facing a big problem of drop out. The rainbow of education remains uncomplete, if the education gets stopped in-between due

Biosc Biotech Res Comm P-ISSN: 0974-6455 E-ISSN: 2321-4007

\section{crossef}

Identifiers and Pagination

Year: 2021 Vol: 14 No (5) Special Issue

Pages: $36-39$

This is an open access article under Creative

Commons License Attribn 4.0 Intl (CC-BY).

DOI: $h t t p: / / d x$.doi.org/10.21786/bbrc/14.5/7 to failure. Preschool, primary, middle school, secondary, junior college and then the senior college, these are steps students climb to get a graduation degree. Many times, student selects a course for graduation because job openings in that domain are high or it's a boom, but not able to survive a long. Lack of interest or poor skills and background of the subject makes students fail in those weak subjects. Allowed to keep terms is the tag get attached with those who fails in the performance during the examination.

This term introduced to the students when they entered the first year of their undergraduate program. To avoid it only way is to take preventive measures. Educational institutes never prefer drop outs, which occurs due to more than three ATKT in one academic year. In this COVIDE-19 pandemic, mobile learning helped learners a lot to go through their lesions and continue their study. ICT (Information Communication Technology) plays a crucial role here; multimedia base learning and online platforms helps such slow learners to learn at their own speed and place.

This study for early detection of prone to failure using machine learning techniques, which helps the student to check his performance using the first semester

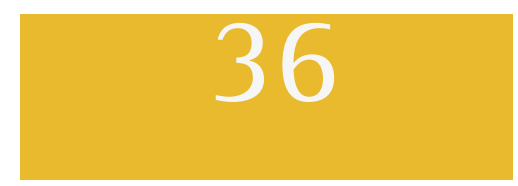


performance which enable them to prepare well in the study if get classified as a poor student. The paper organized in 7 sections. Section 2 gives literature review. This section gives base for further study in this topic. Section 3 clears the objective of the study. Section 4 talks about research methods used in study. Section 5 presents outcomes as results and conclusion Section 6 stands for future scope. Section 7 gives references.

Literature Review: S. B. Kotsiantis (2003) applied six machine learning algorithms namely Naive Bayes, C4.5, Back Propagation, Sequential Minimal Optimization, 3NN, Maximum Likelihood Estimation on data to predict drop out student and performed a comparative study of those algorithm found that Naive Bayes prove the best option. But, in 2018 V. L. Miguéisa proposes a model for classification using data mining techniques, that predicts students' overall academic performance based on the information available at the end of the first year of the students' academic path at the University. The results show that the information available at the end of the first year was sufficient to develop a solid model for a student's performance prediction, the study shows poor performance of Naive Bayes.

It shows a contradiction among the two studies with same algorithms. It's possible, since data uses for these two studies came from two different domains and variables considered also different. Lucas D Ferreira (2018) compare different Machine Learning algorithms for the detection of students' Learning Styles according to the Felder-Silverman Learning Styles model. A dataset of 105 students used here with 252 learning objects during 12 months was extracted from Moodle learning platform. Classification techniques like Naive Bayes, Multilayer Perceptron, Instance-based Learning, and Decision Tree J48 were used. Multiple algorithms required for prediction due to multi-dimensionalities of data. To handle unbalanced datasets Weka's "Class Balancer" algorithm for data preparation and pre-processing was used.

The results demonstrated that the Bayesian learning paradigm has the best performance in the dimensions of processing and organization, whereas the instancebased learning paradigm had the best performance in dimensions of input and perception. Pre-processing technique of class balancing selection \&t the instance filtering process help to improve the results. In the same path, Ahmed Mueen (2016) designed an Educational Data Mining model to predict academic performance based on their academic record and forum participation. Naïve Bayes, Neural Network, and Decision Tree data mining classification algorithms were used on the dataset. It was observed that Naïve Bayes classifier outperforms other two classifiers by achieving overall prediction accuracy of $86 \%$.

Present study helps to find prone to failure student's using classification techniques from machine learning environment. Multiclass classification classifies students into three classes academic success on an undergraduate programme using machine-learning techniques. The study addresses a gap in the literature by applying machine-learning techniques which can capture nonlinear relationships present in real life data. Also, the estimated relationship is used for prediction.

Objective: To perform early detection of prone to failure using machine learning techniques.

\section{METHODOLOGY}

Primary data collect from educational institute base in Mumbai, Kirti M. Doongursee College. Total samples used under study size 300 . Missing values and imbalance data set are the two major problems normally faced in real time data. Less number of failure students can create input bias. Imbalance-learn library from python takes care of it. Features considered for the study was seven subject students learn in the first semester of academic year in Bachelor of Computer Science Course, internal assessment records also considered under this study to evaluate the final outcome, programming skill of the students judged with three practical components. Total 17 input variable used under the study. The three output classes represent poor performing students who achieves less than 40 percentage at the end of semester one, another class belongs to those who secure more than 40 but less than 60 percentage known as average students, third class represents good students indicates marks more than 60 percentage.

\section{Figure 1: Interface of Dataset}

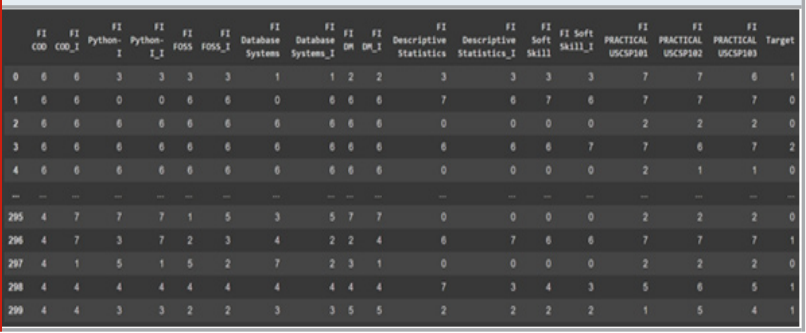

Classification algorithms Naive Bayes classifiers, Logistic Regression used to study the proposed work and come up with multilevel classification using supervised machine learning approach. This approach divides the data into two parts training set and testing set. Since the records considered for this study are less in number so 33 percent data used for testing. The experiment performed with machine learning techniques using colab environment and python libraries for data analysis.

- Gaussian Naïve Bayes: It is one of the three variants based on the Bayes theorem, where probability plays crucial role for classification of data. Conditional probability helps to predict unseen data into the appropriate class. It's proved as a fast algorithm for small set of variables. Its performance well for multiclass classification compared to logistic regression for small size of training sets with categorical data. Gaussian Naïve Bayes assumes that features used for study always follows normal 
distribution. Only two options for parameter tunning considered smoothing values to avoid zero frequency problem and for prior probability, which was set as None indicates no prior probability values considered. $\mathrm{P}(\mathrm{c} \mid \mathrm{X})=(\mathrm{x} 1 \mid \mathrm{c}) \mathrm{x}(\mathrm{x} 2 \mid \mathrm{c}) \mathrm{x} \ldots \ldots \ldots(\mathrm{xn} \mid \mathrm{c})$ $\mathrm{x} P(\mathrm{c})$ GaussianNB(priors=None, var_smoothing $=1 \mathrm{e}-$ 09)

- Logistic Regression: Its an algorithm best suited for the categorical data and belongs to the family of supervised learning algorithms. Three classes considered for the target thus, logistic regression preferred. If need is to classify the data in more than three classes logistic regression proves better choice. Thresholding is the key behind this algorithm which classifies the data by mapping with probability. Overfitting handled with L2 regularization and solver used for fitting the model is lbfgs. LogisticRegression(C=1.0, class_weight=None, dual=False, fit_intercept=True, intercept_scaling $=1$, 11_ratio=None, max_iter=100,multi_class='ovr', $\mathrm{n} \_$jobs $=$None, penalty $=$'l2', random_state $=$None, solver $=$ 'lbfgs', tol $=0.0001$, verbose $=0$, warm start=False)

\section{RESULTS AND CONCLUSION}

The total instances considered for the study was 300 . Distinctly selected attributes 17 . Target classes three namely Poor Performer, Average Student, Excellent Candidates. Data split into two parts training set and testing set. The training set contains 201 records while testing was done on remaining 99 records.

Table 1. Confusion Matrix of Logistic Regression

\begin{tabular}{|l|c|c|}
\hline 10 & 1 & 0 \\
\hline 19 & 35 & 5 \\
\hline 0 & 0 & 29 \\
\hline
\end{tabular}

Table 2. Confusion Matrix of Naïve Bayes

\begin{tabular}{|c|c|c|}
\hline 10 & 1 & 0 \\
\hline 15 & 43 & 1 \\
\hline 0 & 1 & 28 \\
\hline
\end{tabular}

Table 3. Confusion Matrix and classification report of Logistic Regression

\begin{tabular}{|l|c|c|c|c|}
\hline $\begin{array}{l}\text { Logistic } \\
\text { Regression }\end{array}$ & precision & recall & f1-score & support \\
\hline Poor-0 & 0.34 & 0.91 & 0.5 & 11 \\
\hline Averge-1 & 0.97 & 0.59 & 0.74 & 59 \\
\hline Good-2 & 0.85 & 1 & 0.92 & 29 \\
\hline accuracy & & & 0.75 & 99 \\
\hline macro avg & 0.72 & 0.83 & 0.72 & 99 \\
\hline weighted avg & 0.87 & 0.75 & 0.76 & 99 \\
\hline
\end{tabular}

Table 4. Confusion Matrix and classification report of Naïve Bayes

\begin{tabular}{|l|c|c|c|c|}
\hline Naïve Bayes & precision & recall & f1-score & support \\
\hline Poor-0 & 0.4 & 0.91 & 0.56 & 11 \\
\hline Average-1 & 0.96 & 0.73 & 0.83 & 59 \\
\hline Good-2 & 0.97 & 0.97 & 0.97 & 29 \\
\hline accuracy & & & 0.82 & 99 \\
\hline macro avg & 0.77 & 0.87 & 0.78 & 99 \\
\hline weighted avg & 0.9 & 0.82 & 0.84 & 99 \\
\hline
\end{tabular}

As per table 1 logistic regression and table2 naïve bayes out of 11 poor learner 10 were correctly classified but one record was misclassified by the both classifiers. This gives recall 91\%. Precision was calculated for Naïve Bayes for poor performer where total 25 records detected as poor learner but out of 25 only 10 was actually belong to the class poor learner, which takes precision value to $4 \%$.

Results indicates Logistic regression gives results 75\% while the Naïve Bayes algorithms shows $82 \%$ of accuracy, which is comparatively better.

Further Scope: Though the Naïve Bayes algorithms shows 82 percent of accuracy. This analysis accepts the fact that the only first semester grades are not sufficient to analyse student performance. The study can be extended to classify student performance not only on basis of his academic performance but considering his demographics details as well. Which may give significant contribution towards early prediction of prone to failure students.

\section{REFERENCES}

Ahmed Mueen, Bassam Zafar, Umar Manzoor, "Modeling and Predicting Students' Academic Performance Using Data Mining Techniques", I.J. Modern Education and Computer Science, 2016, 11, 36-42, DOI: 10.5815/ ijmecs.2016.11.05

Dr. Vaishali S. Parsania, Dr. N. N. Jani, Navneet H Bhalodiya, "Applying Naïve bayes, BayesNet, PART, JRip and OneR Algorithms on Hypothyroid Database for Comparative Analysis," International Journal of Darshan Institute on Engineering Research and Emerging Technology Vol. 3, No. 1, 2014, pp. 60-64, ISSN 2320-7590

Lucas D Ferreira, Gabriel Spadon, Andr'e CPLF Carvalho, Jose F Rodrigues-Jr, "A comparative analysis of the automatic modeling of Learning Styles through Machine Learning techniques”, 978-1-5386-1174-6/18 @2018 IEEE

Prabha Siddhesh Kadam, "Approach of Undergraduates towards Use of ICT in Education.," International Journal of Advance and Innovative Research, volume 6, Issue 2(XIV): April-June,2019, ISSN 2394-7780 
Prabha Siddhesh Kadam, "Effects of multimedia on learning skills of Kids (2-5 yrs.)”, IOSR Journal of Engineering (IOSRJEN) ISSN (e): 2250-3021, ISSN (p): 2278-8719 PP 20-24

S. B. Kotsiantis, C. J. Pierrakeas, and P. E. Pintelas, "Preventing Student Dropout in Distance Learning Using Machine Learning techniques”, Conference Paper in Lecture Notes in Computer Science September 2003 DOI: 10.1007/978-3-540-45226-3_37•

V. L. Miguéisa, Ana Freitasb, Paulo J. V. Garciab, André Silva "Early segmentation of students according to their academic performance: A predictive modeling approach” , Decision Support Systems 115 (2018) 36-51, 0167-9236/ ๑ 2018 Elsevier B. V. 